\title{
DESIGN ANALYSIS AND CONSTRUCTION OF ENERGY HARVESTING COAXIAL HELICOPTER
}

\author{
Anvinder Singh, Varun Sharma \\ ECE Department, MSIT New Delhi, India \\ E-mails: anvinder_singh26@yahoo.co.in; 91varun.sharma@gmail.com (corresponding author)
}

Received 18 September 2013; accepted 20 November 2013

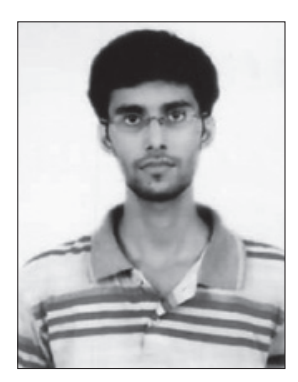

Anvinder SINGH, Eng.

Education: 2009-2013 B. Tech (Eng.) degree in Electronics and Communication, GGS

Indraprastha University, Delhi.

Affiliations and functions: Feb.-Aug. 2012 trainee at solid state physics laboratory

(DRDO), worked on characterisation of thin film and ceramic piezoelectric materials.

Research interest: energy harvesting by piezoelectric materials and advanced designing of coaxial rotor helicopters.

Publications: author of two conference publications.

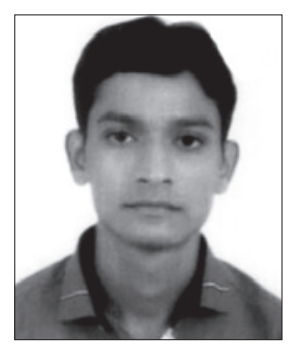
Varun SHARMA, Eng.
Education: 2009-2013 B. Tech (Eng.) degree in Electronics and Communication, GGS Indraprastha University, Delhi.
Affiliations and functions: July-August 2012 trainee at Alcatel-Lucent worked on embedded systems; September-June 2013 worked on project, 'Intelligent Flying
Vehicle', based on embedded systems and wireless transmission.
Research interest: amalgamation of embedded systems and VLSI with piezoelectric materials and advanced mechanics of UAVs and coaxial rotor helicopters.
Publications: one poster presentation at conference.

\begin{abstract}
With the growing need for technology, the tendency for errors has increased many times, which often results in loss of human lives. Our main aim of this paper is to show the implementation of a coaxial rotor aerial vehicle that can be controlled by a radio frequency transmitter. The helicopter is capable of manoeuvring in an area where real helicopters cannot. The area could be a flooded region, a place hit by an earthquake, or a building on fire. The main aim is to transmit video of that place to a base station by the camera attached to the helicopter. Various factors required to make a safe and successful coaxial helicopter are discussed and extensive flight testing proves that this flying machine is better in efficiency and performance than a traditional single rotor aerial vehicle. The relation of flight parameters like torque, induced power, $\mathrm{rpm}$, pitch, and total power are discussed. A piezoelectric sensor is used to determine the vibrations occurring in the body so that they can be minimised. A successful attempt to convert the vibrations into a charge by piezoelectric energy harvesters is made.
\end{abstract}

Keywords: piezoelectric transducer, lift force, characterisation, cantilever, 'C' rating, flight testing.

\section{Introduction}

A tremendous amount of work has been done on multi-rotor helicopters and single rotor helicopters, and each type has its own benefits and drawbacks. Here we open a new horizon to work on coaxial rotor helicopters with a new approach. There is a growing need of CRAV over SRAV on the basis of performance, sta- bility, and other flight parameters. We will also discuss the use of piezoelectric sensors and transducers in storing a charge. The use of vibration sensors is a new approach and it has certainly helped in improving the quality of flight. The use of piezoelectric transducers on the other hand opens horizons for a new concept of flying the vehicle with the energy generated from 
the vehicle itself, i.e. by vibrations, heat, and mechanical force (Narkiewich, Pietrucha 1998). Other forms of energy harvesting techniques such as solar or electromagnetic forms can be amalgamated with piezoelectric technology to improve the flight dependence of the vehicle on batteries, etc. (Kymissis et al. 1998; Roundy 2005). Flight testing has been an important factor in evaluating the performance of this vehicle to avoid ambiguities in transmission and reception of signals at the frequency used $(72 \mathrm{MHz})$.

\section{Coaxial vs. single rotor vehicle}

A coaxial rotor consists of one rotor above the other, rotating in the opposite direction to cancel the gyroscopic force. In general, the bottom rotor rotates in a clockwise direction, and the top rotor rotates in an anticlockwise direction. It implements the principle of compensation of reactive moments about the axis of direction of rotation. Since the motion of the gears of both motors is independent of each other, the thrust on each of them can be varied independently (Lupashin et al. 2011). When the upper rotor is made to rotate fast during flight, it allows the lower rotor to absorb more air, thereby decreasing the power needed to develop lift as compared with a single rotor helicopter. Comparing the efficiency, we found that the CRAV was $80-85 \%$ efficient, whereas the SRAV was $70-76 \%$ efficient. It is worth noting that the CRAV required 10-15\% less power than the SRAV since the latter one needs a tail rotor, which not only uses power but also adds to the weight of the vehicle. The tail rotor adds to the alternate side force, and the coaxial design offers respectable manoeuvrability. On comparing the hovering and vertical climb for both the vehicles simultaneously during the flight tests, we found that the CRAV was easier to control.

The other factors contributing to better stability and control are lack of relation between change in directional and lateral control, yaw moment, and change in collective pitch, but all of this comes at a cost.

It is the absence of a tail rotor that presents limitless advantages to the CRAV. Both vehicles were made to complete a set of tasks in the air, and it was found that the CRAV has a smaller turning radius, and the SRAV collided with a pole while turning. The blade length of both the vehicles is the same, but in the case of the CRAV, we must maintain a minimum distance between the rotor blades to avoid abrasion or collision between them. Figure 1 shows the CRAV during flight; a closer look at the CRAV gives an idea about the gear system and camera used.

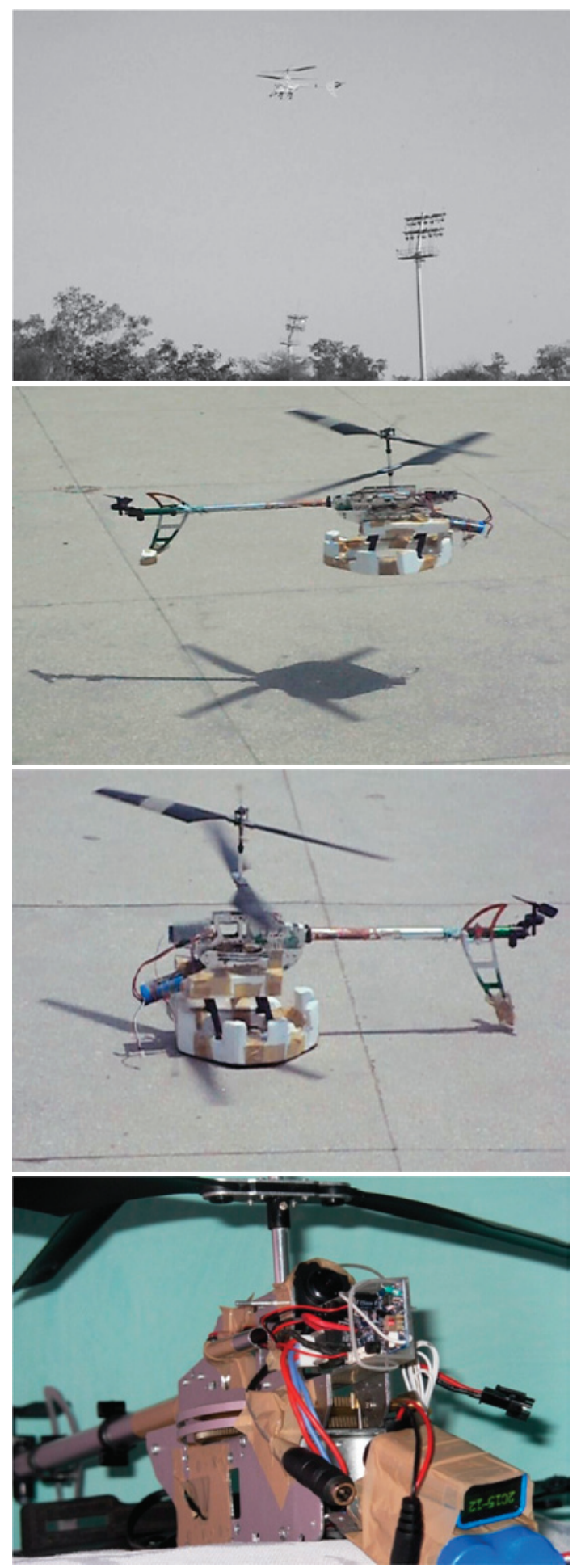

Fig. 1. A close look at the coaxial rotor aerial vehicle

\section{Design analysis}

Designing a coaxial rotor helicopter is more difficult than designing a quad copter because it does not have rotors on all ends to provide stability and controllability (Mussial et al. 2001). Quad copters can be designed using a square shape, which is considered to be efficient for crash landing, or it can be designed using an $X$ shape to provide better manoeuvrability. Our approach while 
designing the CRAV was to minimise the wastage of errors and resources. The first priority was the chassis because a lot of factors depend on its weight, aerodynamic design, and strength (Naido et al. 2011; Bermes et al. 2011). The flight control board should be chosen next as per the requirement of the helicopter (number of motors, ports used for sensors, future expandability). The next step is to estimate the weight of other components like ESC, motors, battery, propellers, payload (Cai et al. 2005), sensors, and receiver so that the configuration of the motors can be decided (Naido et al. 2011). Next we can decide on the type of battery to be used (Fig. 2).

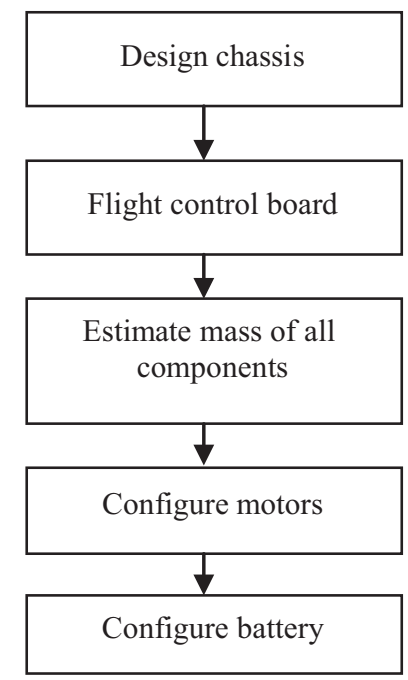

Fig. 2. Design cycle of coaxial rotor helicopter

Our initial theoretical design included a chassis with a coating of silicon in order to withstand high temperatures (up to $1400{ }^{\circ} \mathrm{C}$ ) along with wire rope isolators to minimise vibrations. A chassis coated with plasma-ruggedised solution can withstand both high and low temperatures, but it is costly. An aluminium chassis with conformal coating was ultimately designed to keep the weight to a minimum, and it proved to be stable and controllable during flight testing, although a carbon fibre chassis has also come up as a viable option.

\section{A. Flight control board:}

Three flight control boards were chosen initially, i.e. Arduino UNO, Mega and Pro (Cai et al. 2005). Based on the analogue and digital pins, memory available as per the requirement of the sensors, and receiver to be attached to the Arduino, we decided to go with the Mega since we wish to expand it in the near future. Styrofoam was used to cover the FCB to protect the internal circuitry in case the helicopter crashes. An external piezoelectric gyroscope and accelerometers were used, but many other types of equipment like a magnetometer and GPS are lacking (Cai et al. 2008).

The use of piezoelectric gyros made the flight control sustainable due to their lack of sensitivity to tem- perature effects while flying in sunlight. Figure 3 shows the connections of the propellers, ESC, battery, receiver, accelerometer, and motor with the Arduino.

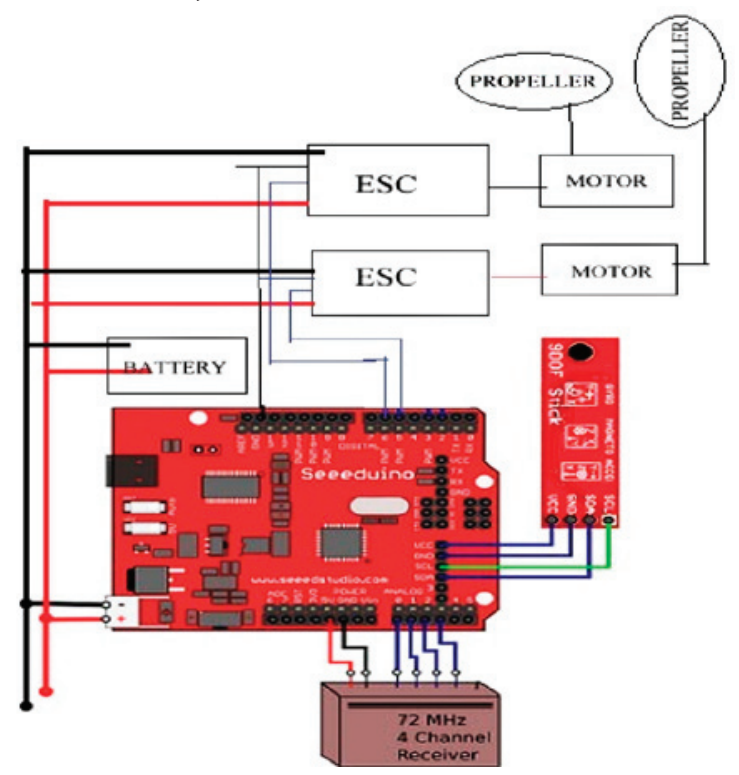

Fig. 3. Arduino connections with the other important components

\section{B. Motors:}

Since the motors decide the efficiency of the systems, they must be tested beforehand to avoid damping performance. We have used outrunner brushless motors because our main aim of flight is to make the CRAV hover at a place to take clear videos. It does not need a suppression capacitor in order to avoid electrical noise coming from the body. The ESC attached to the motor converts the 3-phase AC into DC. They rotate large propellers at low $\mathrm{rpm}$ rather than rotating small propellers at high $\mathrm{rpm}$.

\section{Battery:}

Li-ion batteries do not cut off the supply to the circuit even if the voltage drops to a level less than the threshold, they generate a lot of power at once, and they have a soft cut-off of up to $20-22^{\circ} \mathrm{C}$. Our concern was the ' $\mathrm{C}$ '(Capacity) rating of the battery, which is an indication of its efficiency and depicts the maximum safe continuous discharge rate of a pack. Our battery is $2 \mathrm{~S}$, which means there are two cells in series, and each cell is of $3.7 \mathrm{~V}$. The cells in series give us the voltage, and the voltage of the battery is $7.4 \mathrm{~V}$. The battery is $2500 \mathrm{mAh}$ with a rating of $10 \mathrm{C}$, so it can pull a maximum load of up to $(2500 / 60) \mathrm{mAh}$ per minute $=41.67 \mathrm{mAh}$ per minute.

The $\mathrm{C}$ rating in this case is $10 \mathrm{C}$, and therefore $41.67^{\star} 10 \mathrm{C}=416.7 \mathrm{mAh}$ of draw per minute divided by the pack's capacity $(2500 \mathrm{~mA})=6$ minutes. The battery is completely charged if the voltage reaches $4.2 \mathrm{~V}$ for a cell of 3.7 V. For this case, the battery is charged when it reaches $8.4 \mathrm{~V}$. Overcharging leads to nothing but ruining the battery pack. 


\section{Minimising errors:}

In our design we used a thermocol base, which helped to minimise the vibrations towards the base and to provide better support for the stability of the CRAV. In coaxial rotor helicopters, one blade follows the other one usually 180 degrees later (Musial et al. 2001). If they are balanced (neither higher nor lower), then the rotor blades track properly, and this helps to prevent vibrations and lowers the chance of a crash. The centre of gravity was kept ahead of the shaft because in practical flight conditions it is better to have the CRAV slightly heavy at the nose since it helps in forward flight.

\section{Conditions for hover}

The nominal speed:

$\mathrm{N}_{\mathrm{n}}=\mathrm{V} \times \mathrm{KV}=7.4^{\star} 2400=17,760 \mathrm{rpm}$.

Actual motor speed: $\mathrm{Na}=\eta \times \mathrm{V} \times \mathrm{KV}=0.8^{\star} 7.4^{\star} 2,400=$ $14,208 \mathrm{rpm}$.

Due to the use of the gear system, there is also a reduction in the speed of the shafts and rotors.

Actual speed of blades $=\Omega=(\eta \times \mathrm{V} \times \mathrm{KV}) / \mathrm{g}=$ $\left(0.8^{\star} 7.4^{\star} 2,400\right) / 9.8=1,450 \mathrm{rpm}$.

Lift force on a blade $=\mathrm{F}_{\mathrm{L}}=0.5 \times \mathrm{CL} \times \rho \times \mathrm{V}_{\mathrm{b}}^{2} \times \mathrm{A}$, where:

$\mathrm{C}_{\mathrm{L}}=$ Lift coefficient $=1.6$;

$\rho$ : Density of air $=1.29 \mathrm{~kg} / \mathrm{m}^{3}$;

$\mathrm{A}=$ area swept by blades $=0.1963 \mathrm{~m}^{2}$;

$\mathrm{V}_{\mathrm{b}}=$ Velocity of blades.

$\mathrm{V}_{\mathrm{b}}=(\Omega / 60) \times 2 \pi \mathrm{r}=37.959 \mathrm{~m} / \mathrm{s}$,

so $\mathrm{F}_{\mathrm{L}}=0.5 \times 1.6 \times 1.29 \times 0.1963 \times 37.959^{2}=291.896 \mathrm{~N}$,

so the force required for two rotor disks is $292.52 \times 2=$ $583.8 \mathrm{~N}$.

Speed of rotor in hover condition is:

Lift force on two rotor disks = weight of helicopter body. $2 \times 0.5 \times \mathrm{CL} \times \rho \times \mathrm{v}_{\mathrm{b}}^{2} \times \mathrm{A}=\mathrm{W}_{\mathrm{b}}$

We know the weight of the CRAV, which is $583 \mathrm{~g}$ with the thermocol base.

The $\mathrm{V}_{\mathrm{b}}$ thus comes out to be $37.933 \mathrm{~m} / \mathrm{s}$.

So we can conclude that vertical velocity of $\sim 38 \mathrm{~m} / \mathrm{s}$ is necessary to keep the vehicle hovering.

The blade velocity as a function of the rpm of the motor was also determined practically to understand the relationship between them. The plot is shown in figure 4 .

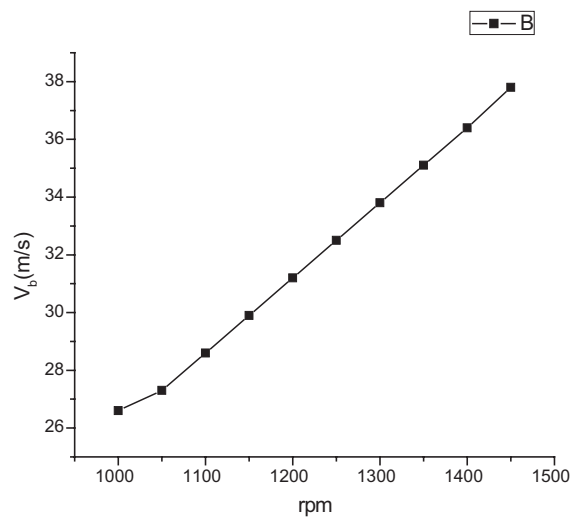

Fig. 4. RPM vs. blade velocity of the CRAV

\section{On-board piezoelectric vibration sensor}

In the laboratory, we manufactured PZT material that was used in the sensor to determine the levels of vibration in the body. After the film was prepared, its ends were etched to make space for electrodes. Characterisation of the sample was done to determine its electrical and dielectric properties, and the sample was found useful to be used as a sensor. The circuit was connected to the Arduino. When vibration is detected, a voltage spike which can go up to $100 \mathrm{~V}$ is produced. The resistance at the analogue terminals prevents the Arduino from being damaged. The positive and negative terminals of the sensor are connected to the analogue terminals of the Arduino. A small $\mathrm{C}$ language code was written wherein one pin is set to low and the other pin acts as a sensory pin. We used both an LED on board the copter and a wireless transmitter and receiver at $433 \mathrm{MHz}$ to send the detected vibrations to the receiver kept with us. The threshold is set via the code, and when the vibrations attain that level, the LED glows and the message 'Threshold reached' appears on the receiver. The LED is handy when FL has not been reached, whereas the LCD is handy during flight. This was helpful in determining whether we should land the CRAV or continue the flight. The following graphs demonstrate the relationship between various parameters (Figs 5-7).

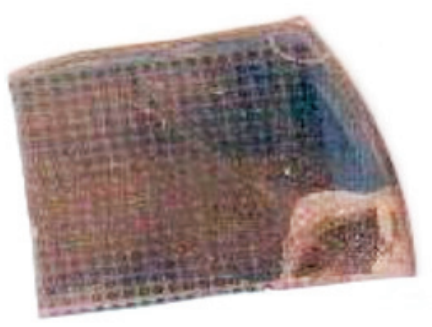

Fig. 5. PZT sample prepared in lab

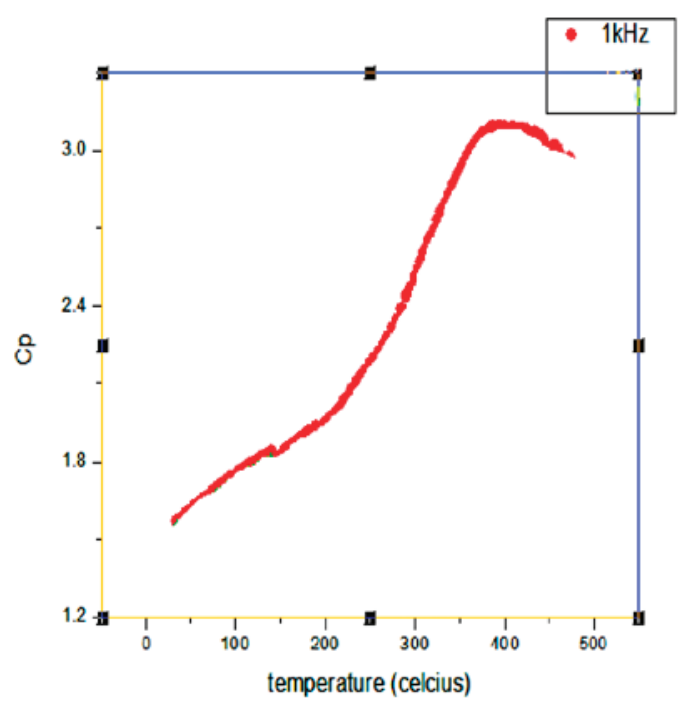

Fig. 6. Plot of parallel capacitance vs. temperature as seen on LCR 


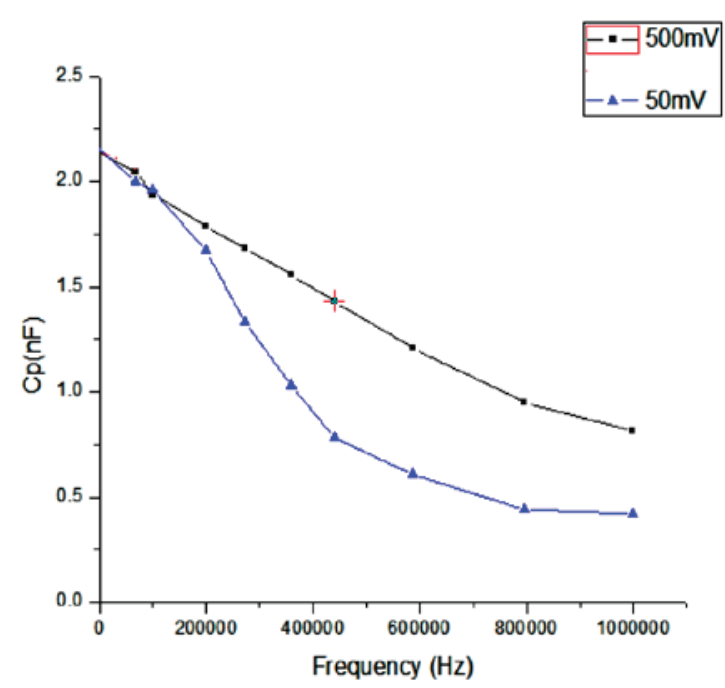

Fig. 7. Parallel capacitance vs. frequency of the prepared PZT

\section{Piezoelectric transducer to harvest vibrational energy}

Another striking feature of this vehicle is the use of a piezoelectric transducer to convert vibrational energy into a charge that can be stored for further use. We used LTC 3588-1 as the medium to convert the vibrational energy. PZT material is attached to the PZT1 and PZT2 pins of the transducer. The input voltage can be varied from 2.7 to $20 \mathrm{~V}$. It must be noted that the frequency of operation of the piezoelectric device must be in the range in which vibrations appear in the chassis.

In our case, since the frequency was around $150 \mathrm{~Hz}$, we chose a device with a frequency range of 80-205 Hz. A capacitor attached to the VIN pin was the energy reservoir for storing charge. It takes vibrations as the input, rectifies the voltage waveform, and stores the harvested energy in a reservoir. Excess power is thrown away with the help of a shunt regulator, and a buck regulator help maintain high efficiency. For the piezoelectric inputs, the time period between various cycles can vary depending on the capacitor value and the source of vibrations.

Capacitor selection: The capacitor should be able to withstand the voltage at VIN at all times, and it must be able to store enough energy to provide output power for the length of time required. The buck must not reach under-voltage lockout, in which case energy transfer to the output would be halted.

$$
\mathrm{P}_{\mathrm{avg}}=1 / 2 \mathrm{CV}^{2} / \Delta \mathrm{t} \text {. }
$$

A V21BL energy-harvesting module has been used as the piezoelectric material for this transducer, which has a displacement of $5.334 \times 10^{-3}$ meters from tip to tip (Ottman et al. 2002). It was mounted in a cantilever manner to the body of the helicopter. A small tip mass can be added to the tip of the cantilever to tune it with the vibrational frequency. In our case we used a tip mass of $2.50 \mathrm{~g}$. A piezoelectric transducer circuit is shown to demonstrate the process better. The pins for the storage capacitor and piezoelectric device are also shown (Fig. 8).

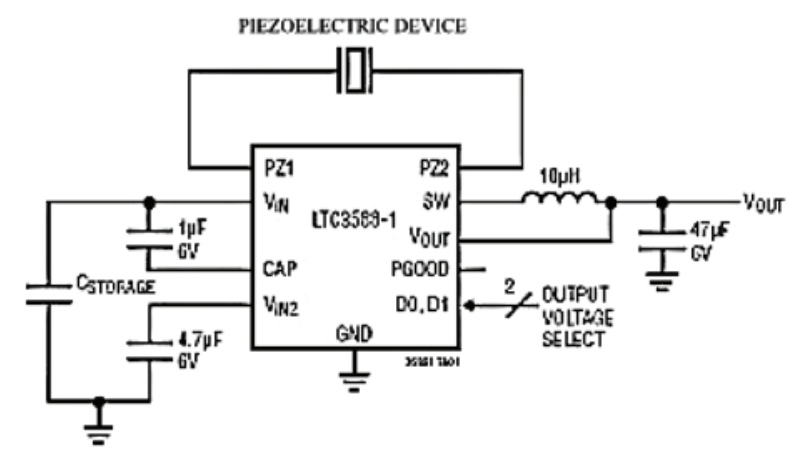

Fig. 8. Piezoelectric transducer circuit

\section{References}

Bermes, C.; Bouabdullah, S.; Schafroth, D., et al. 2011. Design of the autonomous micro helicopter muFly, Mechatronics 21(5): 765-775. http://dx.doi.org/10.1016/j.mechatronics.2010.12.004

Cai, G.; Feng, L.; Chen, B. M., et al. 2008. Systematic design methodology and construction of UAV helicopters, Mechatronics 18(10): 545-558.

http://dx.doi.org/10.1016/j.mechatronics.2008.05.011

Cai, G.; Peng, K.; Chen, B. M., et al. 2005. Design and assembling of a UAV helicopter system, in Proceedings of 5 th International Conference on Control and Automation, 2005, Budapest, Hungary, 697-702.

Kymissis, J.; Kendall, C.; Paradiso, J., et al. 1998. Parasitic power harvesting in shoes, in Second IEEE International Conference on Wearable Computing, 1998.

Lupashin, S.; Schollig, A.; Hehn, M., et al. 2011. The flying machine arena as of 2010, in Proceedings of the IEEE International Conference on Robotics and Automation (ICRA), May, 2011, 2970-2971.

Musial, M.; Brandenburg, U. M.; Hommel, G. 2001. Inexpensive system design: the flying robot "Marvin", in Proceedings of 6 th International UAVs Conference on Unmanned Air Vehicle Systems, Bristol, UK. 23: 1-12.

Naidoo, Y.; Stopforth, R.; Bright, G. 2011. Quad-Rotor Unmanned Aerial Vehicle Helicopter Modelling \& Control. Intech open source publisher.

Narkiewicz, J.; Pietrucha, J. 1998. Reduction of helicopter vibration and noise level by active control technology, Aviation. Scientific Works 3: 83-85.

Ottman, G. K.; Hofmann, H. F.; Bhatt, A. C., et al. 2002. Lesieutre. Adaptive piezoelectric energy harvesting circuit for wireless remote power supply, in IEEE Transactions on Power Electronics, 2002.

Pounds, P.; Mahony, R.; Hynes, P., et al. 2002. Design of a four rotor aerial robot, in Proc. of Australasian Conference on Robotics and Automation, Auckland, 27-29 November, 2002.

Roundy, S. 2005. On the effectiveness of vibration based energy harvesting, Journal of Intelligent Material Systems and Structures 16(10): 809-823.

http://dx.doi.org/10.1177/1045389X05054042 